\title{
AÇÕES DESENVOLVIDAS NA REMAT \\ Revista eletrônica da matemática com vistas à acessibilidade digital ${ }^{\mathrm{i}}$
}

\author{
Luís Henrique Ribeiro da Silva \\ Instituto Federal de Educação, Ciência e Tecnologia do Rio Grande do Sul \\ luis.silva@caxias.ifrs.edu.br \\ Greice da Silva Lorenzzetti Andreis \\ Instituto Federal de Educação, Ciência e Tecnologia do Rio Grande do Sul \\ greice.andreis@caxias.ifrs.edu.br \\ Henri Luiz Fuchs \\ Instituto Federal de Educação, Ciência e Tecnologia do Rio Grande do Sul \\ benrifuchs@bento.ifrs.edu.br \\ Katia Arcaro \\ Instituto Federal de Educação, Ciência e Tecnologia do Rio Grande do Sul \\ katia.arcaro@caxias.ifrs.edu.br \\ Rodrigo Sychocki da Silva \\ Universidade Federal do Rio Grande do Sul \\ sychocki.rodrigo@gmail.com
}

\begin{abstract}
Resumo inclusão digital de usuários com deficiência visual.

Palavras-chave

REMAT. Acessibilidade Digital. Barreiras Tecnológicas. Deficiência Visual.
\end{abstract}

A REMAT: Revista Eletrônica da Matemática (e-ISSN 2447-2689) tem como missão compartilhar práticas educativas e resultados de pesquisas que se relacionam com a matemática. Em busca da consolidação da revista perante a comunidade acadêmica, a equipe editorial da REMAT vem trabalhando no aprimoramento dos processos editoriais, estejam eles relacionados à qualificação do que é produzido ou à acessibilidade digital. A REMAT, editada e publicada pelo Instituto Federal de Educação, Ciência e Tecnologia do Rio Grande do Sul (IFRS) em seu Portal de Periódicos, é um periódico científico eletrônico de acesso livre, com publicação semestral, que visa publicizar produções originais de pesquisadores, sendo estas organizadas em três seções: Matemática em Contextos Técnicos e/ou Tecnológicos, Ensino de Matemática e Matemática Pura e/ou Aplicada. Este artigo apresenta um panorama das ações já desenvolvidas na REMAT no que tange à acessibilidade digital, visando fomentar as discussões sobre a

\section{INTRODUÇÃO}

A crescente difusão das Tecnologias de Informação e Comunicação (TICs) vem transformando gradualmente a nossa sociedade em uma sociedade da informação (NUNES, 2002). Com isso, se faz necessário pensar que cada vez mais pessoas têm acesso à informação. Embora haja um salto quantitativo no acesso ao meio eletrônico, não necessariamente $\mathrm{o}$ aspecto qualitativo cresce em mesma proporção. Em um país no qual aproximadamente 45 milhões de habitantes possuem algum tipo de deficiência (IBGE, 2010), é essencial fazer uma reflexão sobre acessibilidade no meio digital. Teixeira (2014) defende que o ambiente virtual deve ser receptivo a todos os segmentos, e democrático o bastante para atender a todo tipo de interesse coletivo. Conforme Nunes (2002, p. 5):

A Internet é, muitas vezes, mais importante para pessoas com necessidades especiais do que para pessoas sem essas necessidades, pois pode ajudar a ultrapassar as 
barreiras físicas tradicionais. Por exemplo, uma pessoa invisual, utilizando a Internet para fazer compras on-line deixa de estar dependente de terceiros ou de obstáculos físicos.

No entanto, novos desafios surgem, na medida em que se o sítio da loja on-line, no caso do exemplo anterior, não estiver preparado para funcionar com navegadores que lêem o texto da página, utilizando sintetizadores de voz, o utilizador não conseguirá realizar o que pretende.

O relato das ações desenvolvidas na Revista Eletrônica da Matemática (REMAT) apresentado neste artigo visa fomentar as discussões acerca da acessibilidade digital nos periódicos científicos. A acessibilidade de um periódico em formato digital é um compromisso importante de ser assumido, demandando conscientização dos envolvidos nos processos editoriais, bem como disponibilidade de ferramentas adequadas no sistema de editoração. A busca pela acessibilidade digital tem sido um dos objetivos da REMAT desde sua criação e, nessa direção, pretende-se, para as edições futuras a partir de 2018, intensificar as ações relacionadas à acessibilidade digital.

A seguir, com a finalidade de uma contextualização do periódico, faz-se a sua apresentação. Posteriormente, discute-se sobre as ações já desenvolvidas pela REMAT com vistas à acessibilidade digital e sobre o direcionamento do periódico.

\section{HISTÓRICO}

A REMAT teve início em 2015, idealizada por um grupo de docentes do Instituto Federal de Educação, Ciência e Tecnologia do Rio Grande do Sul (IFRS), do Campus Caxias do Sul, com o objetivo de publicizar produções originais com enfoque no Ensino de Matemática, Matemática Aplicada e/ou Matemática Pura, bem como divulgar pesquisas sobre o uso de tecnologias digitais e suas contribuições para estes campos do conhecimento (ANDREIS; SILVA, 2015). Foram publicadas duas edições em 2015. A primeira, totalmente vinculada à Semana Acadêmica do Curso de Licenciatura em Matemática do IFRS, Campus Caxias do Sul, e a segunda com artigos provenientes de diferentes instituições de ensino.

Em 2016, a REMAT passou a apresentar uma seção especial, dedicada à matemática desenvolvida nos Institutos Federais (IFs), tanto a nível de pesquisa científica quanto a nível de práticas de ensino executadas nos cursos técnicos, de graduação e de pós-graduação (ANDREIS; SILVA, 2016). No mesmo ano, foi indexada junto ao Google Acadêmico e ao Diretório de Políticas Editoriais das Revistas Científicas Brasileiras (Diadorim). No final deste mesmo ano, as seções foram novamente reestruturadas, passando a ser:

a. Matemática em Contextos Técnicos e/ou Tecnológicos: artigos apresentando resultados originais, parciais ou finais, de pesquisas científicas desenvolvidas em cursos técnicos e/ou tecnológicos com enfoque na matemática. Incluem-se também práticas de ensino que integrem a matemática com outros componentes curriculares nos cursos em questão.

b. Ensino de Matemática: artigos apresentando resultados originais, parciais ou finais, de pesquisas científicas com enfoque no ensino de matemática, que oportunizem a reflexão sobre a prática docente nos mais diversos níveis e modalidades de ensino.

c. Matemática Pura e/ ou Aplicada: Artigos apresentando pesquisas científicas, parciais ou finais, na área da matemática pura e artigos que apresentem abordagens novas e/ou interessantes para problemas de matemática. Artigos apresentando resultados originais, parciais ou finais, na área da matemática aplicada, com interfaces em outras ciências.

Além disso, ainda em 2016, com a finalidade de fomentar a qualificação do periódico, a equipe editorial foi reestruturada e ampliada, ficando composta por: editor- 
chefe, editor-adjunto, editor-executivo, comissão editorial, conselho editorial consultivo, editores de texto, apoio técnico e avaliadores ad hoc (ANDREIS; SILVA; BOFF, 2016)

A nova constituição do conselho editorial consultivo e do grupo de avaliadores ad hoc visou a atender a uma política de inclusão de pesquisadores de diferentes regiões e instituições do Brasil e do exterior. No início de 2017, o apoio técnico também passou a ter a contribuição de um bolsista para auxílio nos processos de gestão e de divulgação da revista por meio de edital específico da instituição, o qual apoia seus periódicos científicos.

Em janeiro de 2017, o periódico recebeu classificação no Qualis Periódicos, disponibilizada na Plataforma Sucupira da Coordenação de Aperfeiçoamento de Pessoal de Nível Superior (Capes), em cinco áreas de avaliação, conforme exposto na Figura 1.

Figura 1. Classificação da REMAT no quadriênio 2013-2016.

\begin{tabular}{|c|c|c|c|}
\hline \multicolumn{4}{|l|}{ Periódicos } \\
\hline ISSN & Titulo & Área de Avaliação & Classificação \\
\hline $2447-2689$ & REMAT: REVISTA ELETRÔNICA DA MATEMÁTICA & EDUCAÇÃO & c \\
\hline $2447-2689$ & REMAT: REVISTA ELETRÔNICA DA MATEMÁTICA & ENSINO & B3 \\
\hline $2447-2689$ & REMAT: REVISTA ELETRÔNICA DA MATEMÁTICA & INTERDISCIPLINAR & 85 \\
\hline $2447-2689$ & REMAT: REVISTA ELETRÔNICA DA MATEMÁTICA & MATEMÁTICA/PROBABILIDADE E ESTATISTICA & 85 \\
\hline $2447-2689$ & REMAT: REVISTA ELETRÔNICA DA MATEMÁTICA & PSICOLOGIA & 85 \\
\hline
\end{tabular}

Fonte: Capes (2016)

A revista recebe submissões de artigos em fluxo contínuo, que são avaliados por avaliadores ad hoc, de acordo com suas áreas de atuação, em um processo duplamente cego. Os critérios de avaliação observam os seguintes aspectos: relevância e

No Gráfico 1 apresenta-se o número de submissões aceitas e rejeitadas por edição. originalidade, clareza e pertinência dos objetivos, adequação do título ao conteúdo, estrutura coerente, clareza na metodologia, apresentação de resultados e discussão dos mesmos, citações e referências adequadas ao conteúdo. 
Gráfico 1. Número de submissões aceitas e rejeitadas por edição.

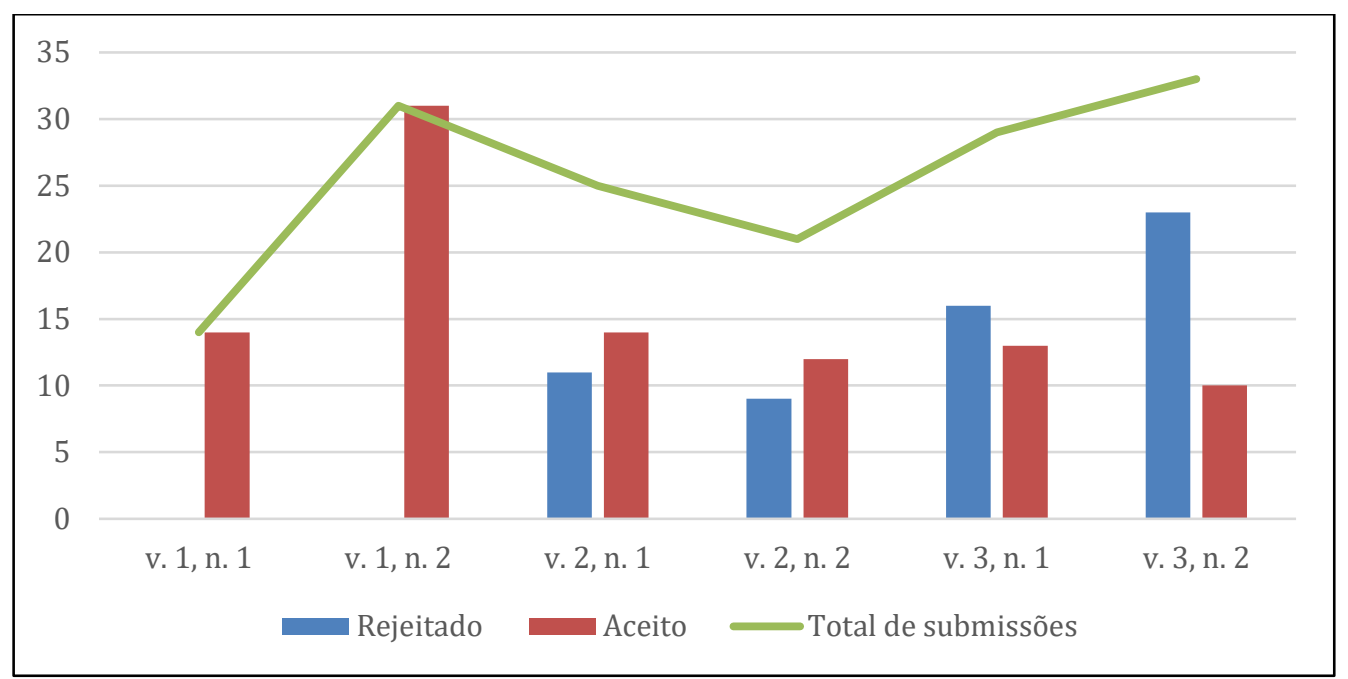

Fonte: REMAT (2017)

\section{ALCANCE DO PERIÓDICO}

De acordo com os relatórios gerados pelo Sistema Eletrônico de Editoração de Revistas (SEER) utilizado pela REMAT, foram contabilizados 7664 acessos aos resumos (na Tabela 1) e 10639 acessos aos arquivos PDF/A de 21 de julho de 2016 a 15 de janeiro de 2018, conforme mostra a Tabela 1. A partir dos resultados nota-se uma crescente busca pelos manuscritos publicados no periódico e sua aceitação na comunidade científica.

Tabela 1. Número de acessos aos resumos e arquivos PDF/A de 21 de julho de 2015 a 15 de janeiro de 2018.

\begin{tabular}{|c|c|c|c|c|c|c|c|c|}
\hline \multirow{2}{*}{ Edições } & \multicolumn{2}{|c|}{$\mathbf{2 0 1 6}$} & \multicolumn{2}{c|}{$\mathbf{2 0 1 7}$} & \multicolumn{2}{c|}{$\mathbf{2 0 1 8}$} & Total de acessos \\
\cline { 2 - 9 } & $\mathbf{R}$ & $\mathbf{P D F} / \mathbf{A}$ & $\mathbf{R}$ & $\mathbf{P D F} / \mathbf{A}$ & $\mathbf{R}$ & $\mathbf{P D F} / \mathbf{A}$ & $\mathbf{R}$ & PDF/A \\
\hline $\begin{array}{c}\text { v. 1, n. } \\
\text { 1, 2015 }\end{array}$ & 94 & 38 & 395 & 493 & 28 & 12 & 517 & $\mathbf{5 4 3}$ \\
\hline $\begin{array}{c}\text { v. 1, n. } \\
\text { 2, 2015 }\end{array}$ & 215 & 284 & 1142 & 1158 & 44 & 42 & 1401 & $\mathbf{1 4 8 4}$ \\
\hline $\begin{array}{c}\text { v. 2, n. } \\
\text { 1, 2016 }\end{array}$ & 712 & 761 & 856 & 2476 & 42 & 63 & 1610 & $\mathbf{3 3 0 0}$ \\
\hline $\begin{array}{c}\text { v. 2, n. } \\
\text { 2, 2016 }\end{array}$ & 516 & 468 & 1604 & 2962 & 30 & 97 & 2150 & $\mathbf{3 5 2 7}$ \\
\hline $\begin{array}{c}\text { v. 3, n. } \\
\text { 1, 2017 }\end{array}$ & - & - & 1625 & 1505 & 30 & 43 & 1655 & $\mathbf{1 5 4 8}$ \\
\hline $\begin{array}{c}\text { v. 3, n. } \\
\text { 2, 2017 }\end{array}$ & - & - & 191 & 116 & 140 & 121 & 331 & $\mathbf{2 3 7}$ \\
\hline Total & $\mathbf{1 5 3 7}$ & $\mathbf{1 5 5 1}$ & $\mathbf{5 8 1 3}$ & $\mathbf{8 7 1 0}$ & $\mathbf{3 1 4}$ & $\mathbf{3 7 8}$ & $\mathbf{7 6 6 4}$ & $\mathbf{1 0 6 3 9}$ \\
\hline
\end{tabular}

$\mathrm{Na}$ Tabela 2 são apresentados os dez artigos mais acessados (arquivo PDF/A), bem como a indicação das edições da revista em que foram publicados. 
Tabela 2. Artigos mais acessados.

\begin{tabular}{|l|c|c|}
\hline \multicolumn{1}{|c|}{ Artigo } & Edição & Acessos \\
\hline A Matemática e o lúdico: ensinando frações através de jogos & v. 2, n. 1 (2016) & 1024 \\
\hline $\begin{array}{l}\text { Cálculo de tarifas e tributos incidentes sobre a conta de energia } \\
\text { elétrica: uma abordagem interdisciplinar no Ensino Médio }\end{array}$ & v. 2, n. 2 (2016) & 1033 \\
\hline $\begin{array}{l}\text { Os PCN e o bloco Tratamento da Informação: algumas } \\
\text { possibilidades teórico-metodológicas para a sala de aula da } \\
\text { Educação Básica }\end{array}$ & v. 2, n. 2 (2016) & 464 \\
\hline $\begin{array}{l}\text { Comparação entre modelos de previsão de demanda: estudo de } \\
\text { caso de um restaurante de comida japonesa }\end{array}$ & v. 2, n. 2 (2016) & 339 \\
\hline $\begin{array}{l}\text { Uma sequência didática para compreender a potenciação e a } \\
\text { radiciação de números complexos }\end{array}$ & v. 2, n. 1 (2016) & 304 \\
\hline $\begin{array}{l}\text { Consumo de energia elétrica: uma atividade interdisciplinar na } \\
\text { Educação de Jovens e Adultos }\end{array}$ & v. 2, n. 1 (2016) & 293 \\
\hline $\begin{array}{l}\text { Geometria Espacial no Ensino Fundamental: construir para } \\
\text { aprender }\end{array}$ & v. 2, n. 1 (2016) & 254 \\
\hline $\begin{array}{l}\text { Instrumentos históricos e o ensino de Matemática: a Régua de } \\
\text { Cálculo Circular e suas contribuições na formação do professor }\end{array}$ & v. 2, n. 2 (2016) & 242 \\
\hline $\begin{array}{l}\text { Exploração de uma situação-problema relacionada à Trigonometria } \\
\text { em cursos de Engenharia }\end{array}$ & v. 3, n. 1 (2017) & 233 \\
\hline $\begin{array}{l}\text { Educação Matemática e Tecnologia: uma análise de discursos } \\
\text { presentes no BOLEMA }\end{array}$ & v. 2, n. 2 (2016) & 232 \\
\hline
\end{tabular}

Fonte: banco de dados estatísticos da REMAT (2018)

Apesar da REMAT ser uma revista científica com seis edições publicadas até o momento, observa-se uma crescente repercussão do periódico. Além do número de acessos aos artigos da REMAT, essa repercussão pode ser evidenciada pela classificação B3 atribuída pela Capes na área de avaliação Ensino. O gráfico da esquerda da
Figura 2 apresenta a distribuição dos 2962 periódicos classificados pela Capes no quadriênio 2013-2016, na área de avaliação Ensino, e o gráfico da direita apresenta a distribuição dos 114 periódicos da área de Matemática, classificados na mesma área de avaliação.

Figura 2. Extratos dos periódicos classificados pela CAPES no quadriênio 2013-2016, na área de avaliação Ensino: todos os periódicos (esquerda); periódicos da área Matemática (direita).

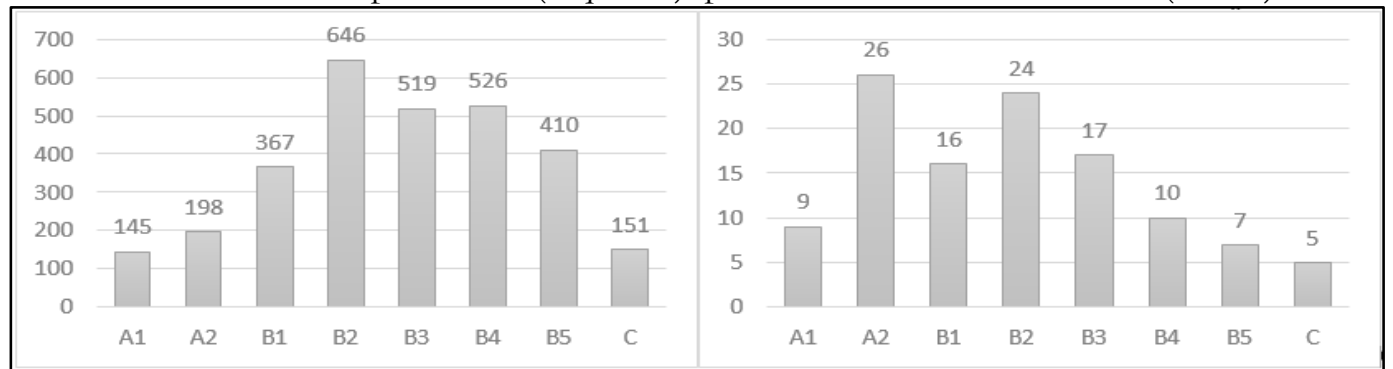

Fonte: Qualis Periódicos (2017) 
O gráfico da esquerda da Figura 3 apresenta a distribuição dos 12 periódicos da área de Matemática criados entre 2013 e 2016 e classificados pela Capes no quadriênio 2013-2016, na área de avaliação Ensino. O gráfico da direita apresenta a distribuição dos cinco periódicos da área de Matemática criados entre 2015 e 2016, classificados na área de avaliação Ensino.

Figura 3. Extratos dos periódicos da área Matemática classificados pela CAPES no quadriênio 2013-2016, na área de avaliação Ensino: criados entre 2013 e 2016 (esquerda); criados entre 2015 e 2016 (direita).

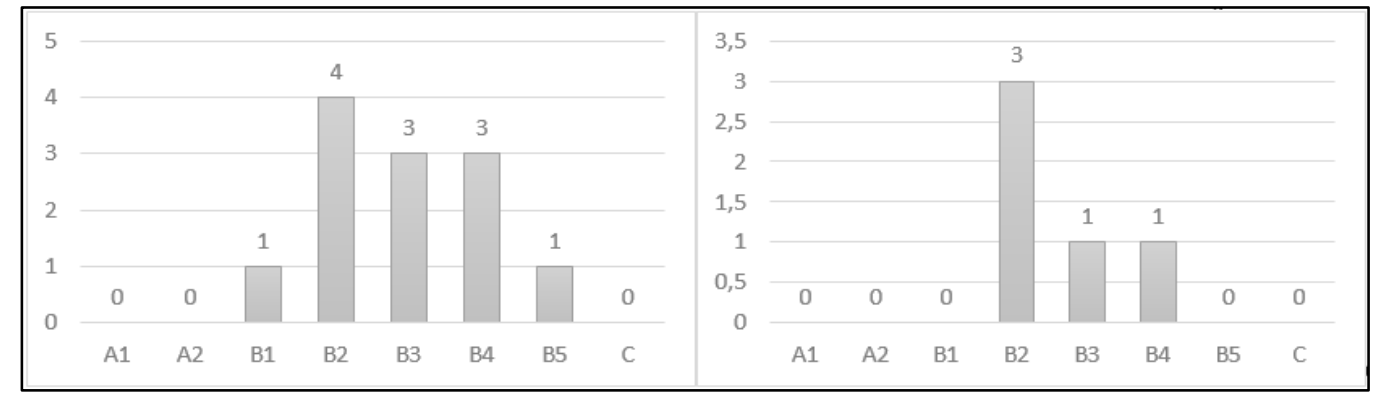

Fonte: Qualis Periódicos (2017)

Com isso, a comunidade de pesquisadores e demais envolvidos no processo de produção e divulgação da REMAT entende que o periódico, além de compartilhar e disseminar conhecimentos científicos, seja também um meio pelo qual novas práticas sobre pensar a matemática e o seu ensino possam ser veiculadas. Com a revista publicada em um website de acesso livre e irrestrito, torna-se possível alcançar, a nível nacional e internacional, professores, estudantes e demais interessados nas temáticas publicadas. Neste cenário, entendese que a partir da reflexão e ação sobre a demanda contemporânea da acessibilidade digital, o raio de alcance da revista aumente de medida. A partir dos gráficos apresentados anteriormente nota-se que o produto REMAT, considerado ainda inacabado, deriva do trabalho dos diversos colaboradores que atuam na direção e processo de aperfeiçoamento do periódico.

\section{AÇÕES DESENVOLVIDAS COM VISTAS À ACESSIBILIDADE DIGITAL}

Entende-se acessibilidade como uma possibilidade e condição de alcance para utilização, com segurança e autonomia, de espaços físicos, equipamentos ou informação por pessoa com deficiência ou com mobilidade reduzida (BRASIL, 2015). Outro conceito importante quando se fala de acessibilidade é o de inclusão que, conforme Sassaki (2009), é o processo pelo qual os sistemas comuns à sociedade são apropriados para toda a diversidade humana. Ainda, define-se barreira como um obstáculo que impede a participação de um indivíduo, ferindo seus direitos à acessibilidade, tal como a liberdade de movimento e o acesso à informação (BRASIL, 2015). Quando se fala em meio digital, no entanto, existem as barreiras tecnológicas que, conforme a Lei $\mathrm{n}^{\circ}$ 13.146 (2015), são aquelas que "[...] dificultam ou impedem o acesso da pessoa com deficiência às tecnologias".

Algumas barreiras tecnológicas em um periódico eletrônico, que ferem o direito à acessibilidade, são percebidas por pessoas com deficiência visual. A deficiência visual "[...] pode ser entendida como a perda ou redução significativa da capacidade visual em ambos os olhos, mesmo após correção, tratamento clínico ou cirúrgico, resultando em cegueira ou baixa visão" (SALTON et al., 2017, p. 27). Com isso exposto, é necessário entender que os recursos utilizados por pessoas cegas e pessoas com baixa visão são distintos. Salton et al. (2017) ressaltam que as pessoas cegas utilizam softwares que fazem a 
leitura da tela e a navegação por meio do teclado. Já pessoas que possuem baixa visão utilizam, além de leitores de tela, ampliadores de tela e recursos de alto contraste (SALTON et al., 2017). No caso dos leitores de tela, é necessário que o documento disponibilizado nos meios eletrônicos esteja configurado de forma correta. Conforme Nunes (2002, p. 6): As pessoas com deficiências visuais (cegos, amblíopes ou daltónicos) são os que, com o actual estado da Web, têm mais dificuldades de acesso, pois a maioria das páginas é fortemente visual. As principais tecnologias utilizadas por estes utilizadores funcionam à base de leitores de ecrã - sintetizadores de vOz ou aparelhos que apresentam o conteúdo das páginas em braille. $\mathrm{Na}$ construção de páginas é importante fornecer alternativas às imagens apresentadas, por forma a não excluir os utilizadores que dependem destas tecnologias.

Nesse sentido, a REMAT tem disponibilizado um modelo editável no formato DOCX que possui estilos préconfigurados. Os estilos, além de promover maior organização da estrutura do texto, permitem que quando o documento for exportado para o formato PDF/A carregue as informações de forma a facilitar o uso de leitor de tela por pessoas cegas. O uso desse recurso, apoiando-se em Salton et al. (2017), permite que o software reconheça uma hierarquia de títulos e subtítulos no documento, já que pessoas cegas não conseguem percebê-las pela diferença do tamanho da fonte ou da cor. Nesse aspecto, este software consegue realizar a leitura de forma contínua e sem interrupções.

Quando se fala em baixa visão, o tipo de fonte utilizada é um fator determinante da acessibilidade digital. Em virtude disso, a REMAT utiliza fontes sem serifa nos artigos, no website e na tipografia do logotipo. $\mathrm{O}$ uso de fontes sem serifa facilita a leitura do usuário. A utilização de fontes com serifa transmite a ideia de algumas letras estarem unidas, devido aos prolongamentos das hastes no final dos caracteres (SALTON et al., 2017). Ainda, Salton et al. (2017) ressaltam que é recomendado evitar a utilização de fontes decoradas e cursivas, não só para facilitar a leitura por pessoas com baixa visão, mas também por pessoas com dificuldades de aprendizagem.

Com relação à acessibilidade digital na parte gráfica, a REMAT utiliza a legenda como método de descrever os gráficos ou imagens, além da descrição no decorrer do manuscrito, logo antes ou logo após a figura. Isso possibilita à pessoa cega uma descrição detalhada da parte gráfica, já que os leitores de tela não têm condições de transformar informações de imagens em áudio. Sobre a acessibilidade na parte gráfica, Salton et al. (2017) ressaltam que a descrição deste conteúdo pode ser feita de três formas: no parágrafo anterior ou logo após a figura ou gráfico; pela legenda deste objeto; ou utilizando uma caixa de texto alternativo. A caixa de texto alternativo permite escrever uma descrição da figura de forma oculta, sem que apareça visualmente, porém, é interpretada e lida pelo leitor de tela (SALTON et al., 2017). Este último procedimento ainda não é adotado pela REMAT.

Relacionado à acessibilidade na navegação no website, a REMAT utiliza um plugin que permite a alteração do tamanho da fonte em tempo real, auxiliando as pessoas com baixa visão. Para este tipo de deficiência visual, uma melhoria a ser feita no periódico é a disponibilização de recursos para a alteração das configurações de contraste.

Com estas implementações, a REMAT procura tornar acessível tanto os meios de navegação no website quanto o acesso universal e individual dos artigos.

\section{CONSIDERAÇÕES FINAIS}

A REMAT, apesar de ser um periódico novo no campo da divulgação científica, tem uma equipe editorial consciente e que constantemente reflete e age sobre as demandas contemporâneas emergentes. Entende-se que a acessibilidade digital seja um tema que mobiliza o interesse de diversas áreas do conhecimento, sendo abordada em pesquisas apresentadas em 
trabalhos de conclusão de cursos de graduação e pós-graduação e também em documentos oficiais. Nesse contexto, nota-se que a visibilidade e o alcance de um periódico demanda que as questões de compartilhamento e socialização do conhecimento recebam destaque. Tais necessidades fazem parte do escopo de trabalho dos editores da REMAT, os quais buscam o aperfeiçoamento dos processos editoriais e na forma em que o material científico é divulgado.

Acredita-se que as ações de aprimoramento realizadas na REMAT sejam consequência do trabalho coletivo, colaborativo, cooperativo e reflexivo do grupo de editores, os quais têm consciência do inacabamento e do caráter evolucionário do processo. Com isso, observa-se que o presente manuscrito tenha alcançado $O$ objetivo de compartilhar junto à comunidade os avanços e o alcance atingidos pela revista, já em um curto espaço de tempo. A primeira avaliação junto a Capes mostra que a REMAT está em uma rota progressiva, e visa ampliar o espectro de alcance e qualificar ainda mais os processos de divulgação e de compartilhamento do conhecimento científico.

Em suma, e com vistas a projeções futuras, o grupo de colaboradores entende que as questões de acessibilidade digital ainda não foram plenamente alcançadas. Com isso, almeja-se que as ações e implementações futuras na revista sejam: uso de links descritivos, uso de links alternativos e uso das ferramentas de alto contraste. As ações mencionadas anteriormente não esgotam as possibilidades de discussões sobre o assunto, e favorecem e fortalecem o cenário descrito, a fim de que outras reflexões e ações ainda possam acontecer.

\section{AGRADECIMENTOS}

Os autores agradecem o apoio financeiro concedido pelo Instituto Federal de Educação, Ciência e Tecnologia do Rio Grande do Sul.

\begin{abstract}
ACTIONS DEVELOPED IN REMAT: REVISTA ELETRÔNICA DA MATEMÁTICA WITH A VIEW TO DIGITAL ACCESSIBILITY

\section{Abstract}

REMAT: Revista Eletrônica da Matemática (e-ISSN 2447-2689) has as mission to share educatives practices and research results that relate with the Math. Looking for the journal consolidation in front of the academic community, REMAT Editorial Staff is working in the editorials processes improvement, whether linked to the qualification of the produced material or linked to the digital accessibility. REMAT, edited and published by Instituto Federal de Educação, Ciência e Tecnologia do Rio Grande do Sul (IFRS) in its Portal of Scientific Journals, is an electronic scientific joumal that offers free access, with semi-annual publication, and aims to publish researchers original productions, being these organized in three sections: Mathematics in Technical and/or Technological Contexts, Mathematics Teaching and Pure and/or Applied Mathematics. This article presents a panorama of actions already developed in REMAT regarding digital accessibility, in order to foster discussions about the digital inclusion of users with visually deficiency.
\end{abstract}

\title{
Keywords
}

REMAT. Digital Accessibility. Technology Barriers. Visual deficiency.

Artigo recebido em 15/01/2018 e aceito para publicação em 18/02/2018

\section{REFERÊNCIAS}

ANDREIS, Greice da Silva Lorenzzetti; SILVA, Rodrigo Sychocki da. Apresentação. Remat, Rio Grande do Sul, v. 1, n. 1, mar. 2015. Semestral. Disponível em: $<$ https://periodicos.ifrs.edu.br/index.php/ REMAT/article/view/1178/1064>. Acesso em: 20 nov. 2017.
ANDREIS, Greice da Silva Lorenzzetti; SILVA, Rodrigo Sychocki da. Apresentação. Remat, Rio Grande do Sul, v. 2, n. 1, mar. 2016. Semestral. Disponível em: $<$ https://periodicos.ifrs.edu.br/index.php/ REMAT/article/view/1287>. Acesso em: 20 nov. 2017. 
ANDREIS, Greice da Silva Lorenzzetti; SILVA, Rodrigo Sychocki da; BOFF, Daiane Scopel. Apresentação. Remat, Rio Grande do Sul, v. 2, n. 2, set. 2016. Semestral. Disponível em: $<$ https://periodicos.ifrs.edu.br/index.php/ REMAT/article/view/1640

>. Acesso em: 20 nov. 2017.

BRASIL. Constituição (2015). Lei $\mathbf{n}^{\circ}$ 13.146, de 6 de julho de 2015. Institui A Lei Brasileira de Inclusão da Pessoa Com Deficiência. Brasília, DF, Disponível em: $<$ http://www.planalto.gov.br/ccivil 03/ at o2015-2018/2015/lei/113146.htm>. Acesso em: 12 out. 2017.

Instituto Brasileiro de Geografia e

Estatística. Censo demográfico 2010: características gerais da população, religião e pessoas com deficiência. Disponível em: $<$ https://biblioteca.ibge.gov.br/pt/bibliotec a-catalogo? view $=$ detalhes\&id $=794>$. Acesso em: 15 jan. 2018.

NUNES, S. S. A Acessibilidade na Internet no Contexto da Sociedade da Informação. 2002. 17 f. Dissertação (Mestrado em Gestão de Informação) - Faculdade de Engenharia. Universidade do Porto, Porto, Portugal,

i Versão revista e ampliada do Trabalho "Ações desenvolvidas na REMAT: revista eletrônica da matemática com vistas à acessibilidade digital" apresentado no evento ABEC MEETING, 6-9, novembro, Curitiba, PR, 2017.
2002. Disponível em: $<$ https://web.fe.up.pt/ mgi01016/is/acessi bilidade.pdf $>$. Acesso em: 15 jan. 2018.

SALTON, B. P.; DALL AGNOL, A.; TURCATTI, A. Manual de Acessibilidade em Documentos Digitais. Bento Gonçalves: Instituto Federal de Educação, Ciência e Tecnologia do Rio Grande do Sul, 2017.

Disponível em: $<$ http://cta.ifrs.edu.br/publicacoes/visu alizar/137>. Acesso em: 15 jan. 2018.

SASSAKI, R. K. Inclusão: acessibilidade no lazer, trabalho e educação. Revista Nacional de Reabilitação, São Paulo, p. 10-16, ano XII, mar./abr. 2009. Disponível em: < https://acessibilidade.ufg.br/up/211/o/SA SSAKI - Acessibilidade.pdf?1473203319>. Acesso em: 20 nov. 2017.

TEIXEIRA, A. P. P. Acessibilidade Digital para a Educação Inclusiva: Desafios e Oportunidades. Diálogo, Canoas, n. 27, p. 97-107, dez. 2014. Disponível em: $<$ https://revistas.unilasalle.edu.br/index.ph $\mathrm{p} /$ Dialogo/article/view/1661>. Acesso em: 12 out. 2017. 